\title{
A Fast Finite Element Model Taking Into Account 3D Effects for the Optimal Design of Micro-Hybrid Starters
}

\author{
Raphael Andreux ${ }^{1,2}$, Julien Fontchastagner ${ }^{1}$, Noureddine Takorabet $^{1}$, and Nicolas Labbe ${ }^{2}$ \\ ${ }^{1}$ Université de Lorraine, GREEN, EA 4366, Vandœuvre-lès-Nancy, F-54500, France \\ ${ }^{2}$ Valeo Electrical Systems, Ville Nouvelle de l'Isle d'Abeau, 38291, St Quentin Fallavier, France
}

\begin{abstract}
This paper presents a fast method to take into account three dimensional effects in reinforced brushed DC motor used as starter in automotive application. Due to their low cost and simplicity, reinforced starters are used in micro-hybrid vehicles. The specific geometry of such devices induces axial component of the flux which cannot be accounted for by 2D models. 3D finite element models are suitable fur this purpose but their excessive need of CPU time complicates their use in an automatic optimization procedure. The authors propose to identify the overhang effects by using an adapted and corrected $2.5 \mathrm{D}$ finite element model which is validated by 3D computations and experiments. Finally, the $2.5 \mathrm{D}$ model is implemented in a multi-objective optimization procedure.
\end{abstract}

Index Terms-Starter, Brushed DC motor, Overhang effects, 2D and 3D Finite Element Method, Global Optimization.

\section{INTRODUCTION}

W ITH the new European standards for fuel economy and reductions in $\mathrm{CO} 2$ emissions [1], many technologies which are more respectful of the environment have emerged in the automotive industry. The technologies have been classified in three levels: full-hybrid, mild-hybrid and micro-hybrid which is also known as stop-start system.

The full hybrid system is able to move the car by using the electric motor for long distance by using the energy stored in the batteries. The rated power of the electric motor comparable to that of the Internal Combustion Engine (ICE). It allows the energy recovery form the wheels to the batteries. The mildHybrid system uses necessarily the electric motor-generator with the ICE and can fulfill the energy recovering function. The rated power of the electric machine is lower up to $10 \mathrm{~kW}$.

The stop-start function consists in stopping a combustion engine when the vehicle pulls to a stop, and restarting it when the driver accelerates. This function can be provided by a starter-generator or even just a reinforced starter. Direct current motors are frequently used for this function. For economic reasons, it may be interesting to make some improvements in the performances of such machines in order to reach the requirements of the micro-hybrid function.

A reinforced starter is a device which contains $12 \mathrm{~V} \mathrm{DC}$ motors that develops about $2 \mathrm{~kW}$ mechanical power in a really compact volume [2], [3]. Therefore, the magnetic circuit is highly saturated. Due to their overhang structure shown on Fig. 1, 3D effects are so important that classical 2D models are not suitable for the modeling of such devices in particularly because of magnetic saturation effects. The 3D modeling of electric machines is necessary in such situation but it is time consuming and is not really adapted for optimization purposes (which is our goal). Different methods have been proposed to take into account the overhang structure by introducing

Manuscript received July XX, 2013; revised September XX, 2013. Corresponding author: J. Fontchastagner (email: julien.fontchastagner@univlorraine.fr). additional coefficients in 2D models [4], [5]. For the yoke stator overhang, an alternate way can be proposed. It consists in performing two finite element simulations in two orthogonal 2D domains and combines the results, leading to a modified $2 \mathrm{D}$ model (it is called a $2.5 \mathrm{D}$ model). In this paper the authors use this idea and perform FE simulations in both $(r, \theta)$ and $(\theta, z)$ domains to determine the corrective parameters of a $2.5 \mathrm{D}$ model more suitable for iterative calculations in an optimization process. The authors also deal with the $2 \mathrm{D}$ equivalent active length for a $2 \mathrm{D}$ field computation when both stator and rotor have significant different lengths.

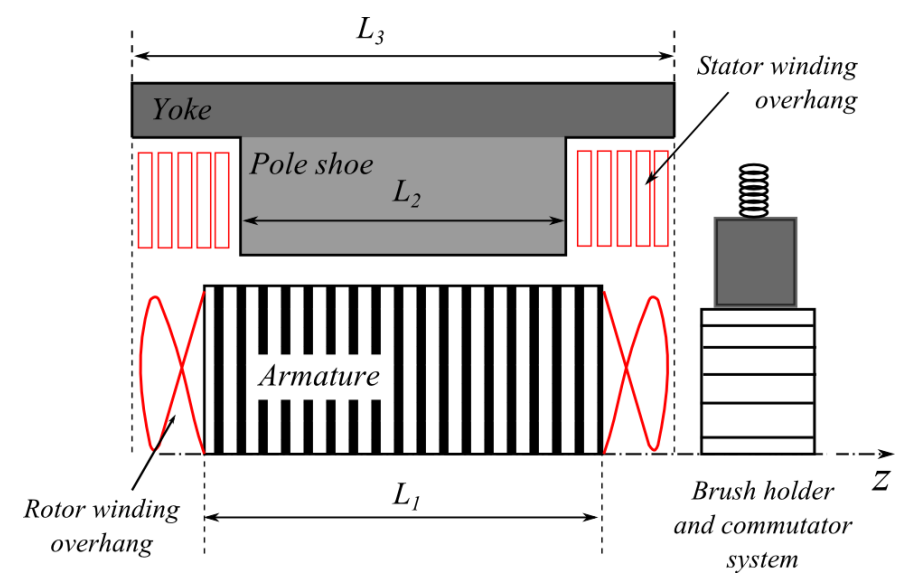

Fig. 1. View of axial ferromagnetic lengths of a wound DC starter

In the first part, the necessity of having a fast and accurate model taking into account two kinds of end effects is highlighted. In the next two sections, two methods are proposed for modeling each effect. A general 2.5D modified model taking into account all overhang effects is presented. It is then validated by comparisons with a 3D model and measurements. Finally, the proposed model is used for solving efficiently some optimization problems corresponding to starters designs examples. 


\section{Problem Statement}

A. Necessity of a fast model for future automatic optimization schemes

The aim of the present work is to provide a fast and efficient tool which can be used in a multi-objective optimization process where, for instance, it would be interesting to determine the Pareto front of the global external volume vs. the electromagnetic torque taking into account the 3D effects. In the literature, end effects are often studied with 3D finite element method for mounted permanent radial magnet [6], [7], or for spoke-type rotor [8] with permanent magnet. In literature, end effects also concern flux switching machines [9], superconducting motors [10] or linear motor [11].

However the authors generally used onerous 3D tools [5], [12], which are CPU time consuming for our purpose. A possible solution would be to use surface response methodologies [13], [14], eventually based on kriging techniques [15], [16]. Such methods are more particularly relevant for monoobjective problems rather than multi-objective ones.

In these circumstances, it is not really appropriate to consider 3D models for our target application. This is why a specific lighter model has been developed and detailed in the following sections.

\section{B. Description of the geometry}

In wound DC motors used as starters, iron axial lengths are different as showed in Fig. 1 to reduce the total length (i.e. the iron pole plus the winding overhang) while ensuring good performances. In our case, the geometry is separated into three parts which have different iron lengths:

1) the axial length $L_{1}$ of the rotor armature;

2) the axial length $L_{2}$ of the pole shoes;

3) the axial length $L_{3}$ of the stator yoke.

Fig. 1 shows that the total external length imposes:

$$
L_{3}=L_{2}+2 \cdot L_{s w h}=L_{1}+2 \cdot L_{r w h}
$$

where $L_{s w h}$ and $L_{r w h}$ respectively represent stator and rotor winding overhang lengths.

The thickness of the yoke is called $e$ as shown in Fig. 2. The aim of our work is to study separately the two overhang effects to provide next the best configuration, e.g. which maximizes the electromagnetic torque in a minimum volume. The two considered effects are described as follow:

- $\mathbf{1}^{\text {st }}$ effect: The yoke hangs over the pole shoes: $L_{3}>L_{2}$; - $2^{\text {nd }}$ effect: The armature hangs over the pole: $L_{2}<L_{1}$.

\section{Insufficiency of a basic 2D model}

A first approach consists in computing the electromagnetic torque, as a function of the supply current, with a static $2 \mathrm{D}$ finite element model representing the median plane of the starter, and neglecting the overhang effects. The active length for torque calculation is assumed to be the armature one, i.e. $L_{2}$. The obtained results are compared to the measured mechanical torque $\Gamma_{\text {mech }}$ of an existing machine and are presented on Fig. 3.

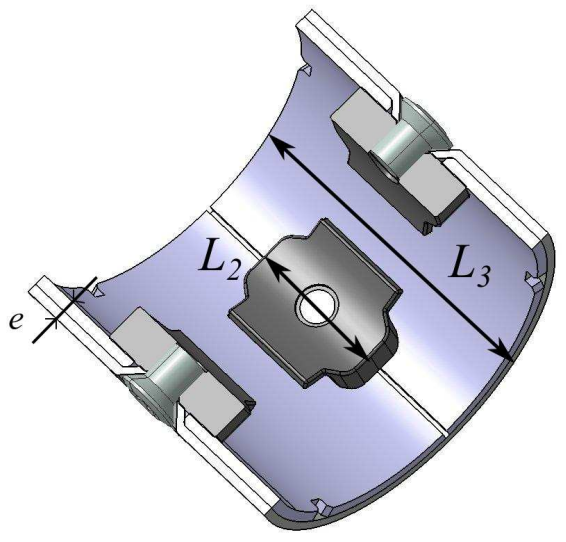

Fig. 2. View of the pole shoes screwed on the yoke.

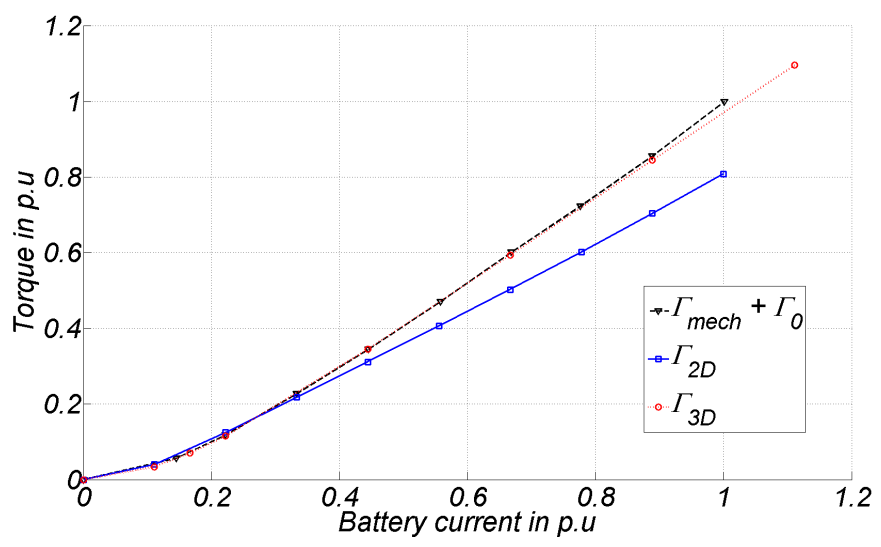

Fig. 3. Comparisons of the torque vs. current expressed in p.u.

During our experiments, the output mechanical torque $\Gamma_{\text {mech }}$ produce by the studied DC motor starter is measured with a torque meter. In such devices the losses are mainly located in the "Brushes-collector" system as friction losses and also in the stator and rotor windings as copper losses. The iron losses can be neglected because the iron volume involved in these losses is very small. For instance, on the prototype used for experiments, core losses represent $2 \%$ of the total power, whereas the copper and mechanical losses represents more than $20 \%$ of the total power.

The main part of the mechanical losses can be considered as only due to the friction of the brushes. Thus, $\Gamma_{0}$, the equivalent torque corresponding the mechanical losses, is constant and equal to the difference between the mechanical torque and the electromagnetic one $\left(\Gamma_{\mathrm{el}}\right)$; and it can be experimentally determined. In this case, if we want to compare the computed electromagnetic torque with measurements, we must use the sum of these two torques according to (2):

$$
\Gamma_{\mathrm{el}}=\Gamma_{\mathrm{mech}}+\Gamma_{0}
$$

It appears that the $2 \mathrm{D}$ model - where $3 \mathrm{D}$ effects are not considered - underestimate significantly the torque when the current increases (nearly 20\%). Of course, a static 3D model is clearly more efficient as it is shown by the red curve. But 
such a model is too heavy, in terms of CPU time for our future target applications.

\section{CONSIDERATION OF THE $1^{\text {ST }}$ EFFECT: CONSTRUCTION OF A 2.5D MODEL}

In this part, only the first effect is studied: the yoke versus pole shoes overhang (i.e. $L_{3}>L_{2}$ ). Thus, the $L_{1}$ and $L_{2}$ lengths are supposed to be equal and only $L_{3}$ differs to be greater than the first two ones.

This overhang structure has a significant influence on the saturation level of the ferromagnetic materials and the flux path in the yoke has a purely three dimensional behavior. A 2D model does not take into account this phenomenon and may give bad results especially if the yoke is highly saturated. The goal is to take into account this phenomenon by adapting the thickness $e^{\prime}$ of the 2D model (Fig. 4a). This fictive increase of the yoke thickness models the part of the flux through the overhang yoke. It is computed according to the principle of equivalence between two models in terms of yoke reluctance. It is described as follows:

1) In the domain \#1, of axial length $L_{1}$, the reluctance of the yoke is computed by:

$$
\mathcal{R}_{1}=\frac{M M F_{1}}{\varphi}=\frac{\int_{C_{1}} \mathbf{H} \cdot \mathbf{d} \mathbf{l}}{L_{1} \int_{\Gamma_{1}} \mathbf{B} \cdot \mathbf{d s}}
$$

where $C_{1}$ is the dashed arc line and $\Gamma_{1}$ the segment both given in Fig. 4a.

2) In the domain \#2 the field calculation is performed in the $(\theta, z)$ domain and consists in calculating the flux distribution on the yoke surface by considering special boundary conditions. The two potentials $A_{1}$ and $A_{2}$ in the boundaries represented by the dashed lines on the Fig. $4 \mathrm{~b}$ are imposed; and two periodic conditions for the remaining boundaries are applied. In this case, the effective thickness $e$ of the yoke represents the third coordinate orthogonal to the domain \#2. The values of the potentials $A_{2}$ and $A_{1}$ used as Dirichlet conditions are determined so that: $e \cdot\left(A_{2}-A_{1}\right)=\varphi$ so that the two calculations are equivalent in terms of flux. The reluctance of the yoke is then computed by:

$$
\mathcal{R}_{2}=\frac{M M F_{2}}{\varphi}=\frac{\int_{C_{2}} \mathbf{H} \cdot \mathbf{d} \mathbf{l}}{e \cdot\left(A_{2}-A_{1}\right)}
$$

3) At this step, the modified thickness $e^{\prime}$ is adapted and computed so that $\mathcal{R}_{2}=\mathcal{R}_{1}$. The principle of the used procedure is given by Fig. 5. A simple fixed point method which converges quickly (around ten iterations corresponding to twenty $2 \mathrm{D}$ computations) is used. In this case, the flux and torque computed by the modified 2D model take into account the 3D effects due to the geometry of the yoke, thus it is abusively called a "2.5D model".

Fig. 6 shows the ratio $e^{\prime} / e$ vs. the ratio $L_{3} / L_{2}$ which is growing and becomes stable when $L_{3}>L_{2}$. This asymptotic behavior is naturally understandable since the flux path in the

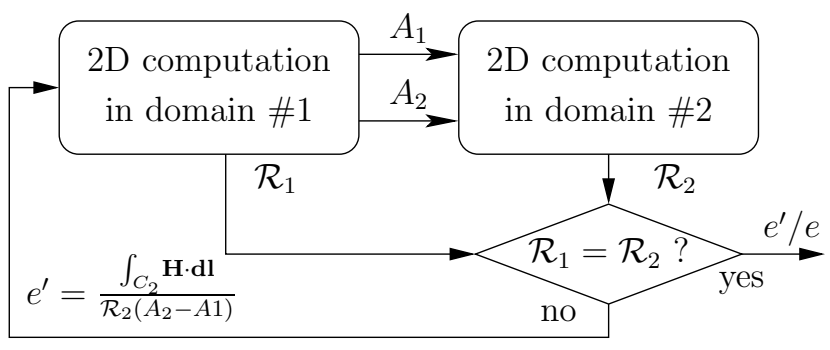

Fig. 5. Scheme principle of the 2.5D model.

yoke is not modified beyond a given value of $L_{3}$. Full 3D finite element calculations have been performed for three different values of the ratio $L_{3} / L_{2}$ in order to validate the $e^{\prime} / e$ ratio values of the 2.5D model. Results of the basic $2 \mathrm{D}$ model and the $2.5 \mathrm{D}$ model are compared with 3D calculations for four different battery currents and displayed on Table I.

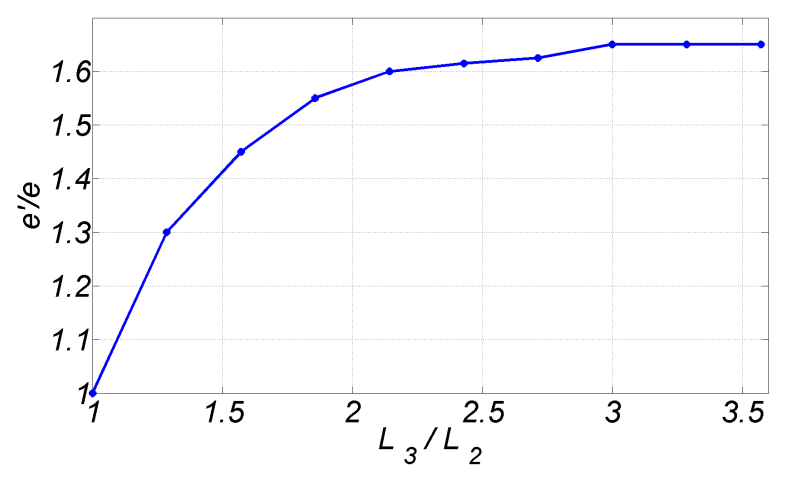

Fig. 6. Results of the 2.5D model $-e^{\prime} / e$ vs. $L_{3} / L_{2}$.

TABLE I

COMPARISON OF THE 2.5D MODEL WITH 3D COMPUTATIONS

\begin{tabular}{c|c|c|c|c}
$\begin{array}{c}L_{3} / L_{2} \\
\text { ratio }\end{array}$ & $\begin{array}{c}e^{\prime} / e \\
\text { ratio }\end{array}$ & $\begin{array}{c}\text { Battery } \\
\text { current }(\mathrm{A})\end{array}$ & $\begin{array}{c}\Delta\left(\Gamma_{2 \mathrm{D}}, \Gamma_{3 \mathrm{D}}\right) \\
(\%)\end{array}$ & $\begin{array}{c}\Delta\left(\Gamma_{2.5 \mathrm{D}}, \Gamma_{3 \mathrm{D}}\right) \\
(\%)\end{array}$ \\
\hline \hline \multirow{3}{*}{1.3} & \multirow{3}{*}{1.3} & 200 & -8.4 & $\mathbf{- 1 . 2}$ \\
& & 400 & -12.1 & $\mathbf{1 . 9}$ \\
& & 600 & -12.8 & $\mathbf{1 . 4}$ \\
& & 800 & -13.0 & $\mathbf{0 . 8}$ \\
\hline \multirow{3}{*}{1.715} & \multirow{3}{*}{1.56} & 200 & -11.4 & $\mathbf{- 1 . 9}$ \\
& & 400 & -19.7 & $\mathbf{- 1 . 0}$ \\
& & 600 & -21.8 & $\mathbf{- 1 . 0}$ \\
& & 800 & -21.9 & $\mathbf{- 0 . 8}$ \\
\hline \multirow{3}{*}{2.29} & \multirow{3}{*}{1.62} & 400 & -12.4 & $\mathbf{- 2 . 5}$ \\
& & 600 & -20.8 & $\mathbf{- 1 . 6}$ \\
& & 800 & -23.2 & $\mathbf{- 1 . 7}$ \\
\hline
\end{tabular}

The given results show that the 2.5D model, which takes into account the fictive thickness of the yoke, is very close to the 3D computations (the relative difference is less than 3\%) whereas the non-modified 2D model underestimate the torque (the relative difference can exceeds $20 \%$ ). 


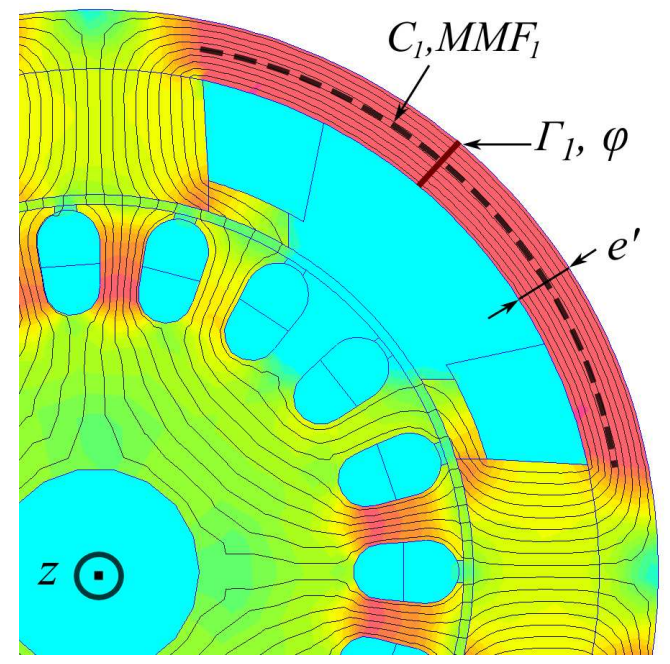

(a) Axial View (domain \#1)

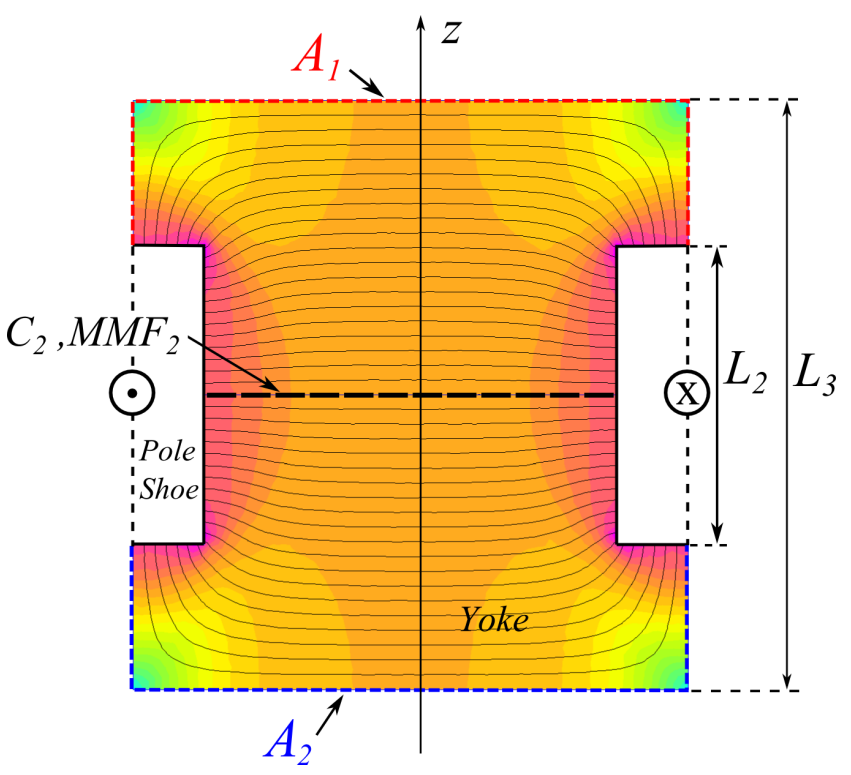

(b) Orthogonal domain (domain \#2)

Fig. 4. Representation of the both used 2D finite element models.

\section{CONSIDERATION OF THE $2^{\text {ND }}$ EFFECT: CONSTRUCTION OF A 2D CORRECTED MODEL (2D $\mathrm{D}_{\mathrm{COR}}$ MODEL)}

In this part, the focus is on the second overhang effect between the armature and the pole shoes. For compactness reasons, the armature is often longer than the pole shoes $\left(L_{1}>L_{2}\right)$ as shown on Fig. 1 In such condition, end effects in axial direction exist and are quite pronounced. Fig. 7 highlights this by displaying the flux density distribution on the armature surface. The entire iron armature surface does not participate in the same manner to the electromechanical conversion. Thus, the real active length of the device should be between $L_{1}$ and $L_{2}$. This length, named $L_{2 \mathrm{D}}$, must be the one used on the 2D finite element model for the torque calculation. In the following, $\xi\left(L_{2} / L_{1}, I\right)$ is defined. It corresponds to the corrective ratio which should be applied to the $L_{1}$ length to obtain the correct length $L_{2 \mathrm{D}}$. This ratio, given in (5) depends on the magnetic saturation (given by the battery current $I$ ) and the $L_{2} / L_{1}$ ratio. It is necessarily strictly between $L_{2} / L_{1}$ and 1 .

$$
L_{2 \mathrm{D}}=\xi\left(L_{2} / L_{1}, I\right) \times L_{1}
$$

The $\xi\left(L_{2} / L_{1}, I\right)$ ratio has been identified thanks to a standard design of experiment (DOE) based on 3D finite element computations. The $L_{1}$ length is fixed and both the $L_{2}$ and $L_{3}$ lengths vary simultaneously $\left(L_{2}=L_{3}\right)$ in order to cover $L_{2} / L_{1}$ in the range $[0.65 ; 1]$. Such bounds values are representative of cases encountered in industrial devices.

In order to determine $\xi\left(L_{2} / L_{1}, I\right)$, the $3 \mathrm{D}$ computations are then compared to a modified $2 \mathrm{D}$ model which takes into account the different rotor and stator axial length by modifying the permeability of the pole shoes. It is called $2 D_{\text {Cor }}$ model for 2D corrected model. Such a model uses the armature length $L_{1}$ as the active length for torque calculations. Thus, a smart way to consider the fact that the length of the pole shoe is smaller than $L_{1}$ is to considered that the pole shoes are composed

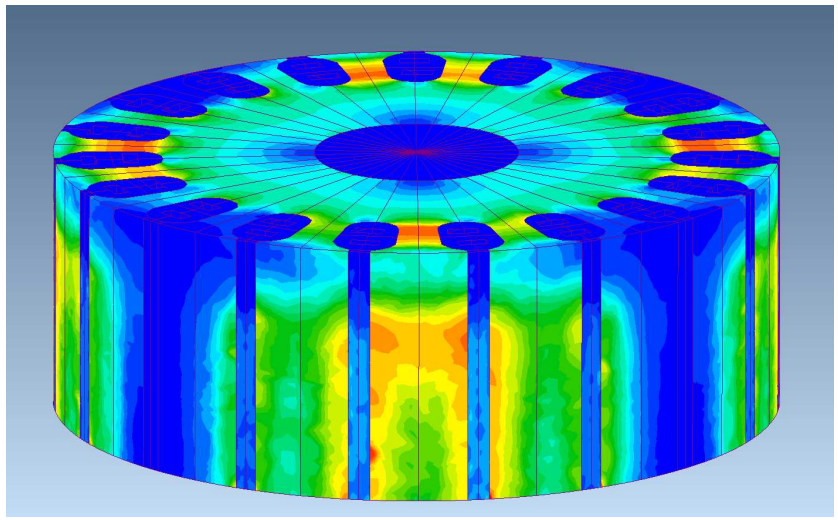

Fig. 7. Induction map in one half armature surface.

by a mix of iron and vacuum. It is expressed in the modified permeability $\mu e q$ used in the $2 \mathrm{D}_{\text {Cor }}$ model as:

$$
\mu_{e q}=\mu_{0}\left(1-\frac{L_{2}}{L_{1}}\right)+\mu_{i} \frac{L_{2}}{L_{1}}
$$

where $\mu_{0}$ and $\mu_{i}$ respectively represent the permeability of the vacuum and the iron. It is also considered that the active length to be used for torque calculation is the longest one (e.g. the length $\left.L_{1}\right)$. Finally, the $\xi\left(L_{2} / L_{1}, I\right)$ coefficient determined by (7) represents the aptitude of the $2 \mathrm{D}$ model to overestimate the torque calculation because of end effect.

$$
\xi\left(L_{2} / L_{1}, I\right)=\frac{\Gamma_{3 \mathrm{D}}(I)}{\Gamma_{2 \mathrm{D}}\left(I, L_{1}, L_{2}\right)}
$$

Results displayed on Fig. 8 (in \%), show that the $\xi\left(L_{2} / L_{1}, I\right)$ ratio decreases with battery current and/or $L_{2} / L_{1}$ ratio. Moreover the end effect is more pronounced for low levels of saturation. After this preliminary step, if the $L_{1}>L_{2}$ effect had to be taken into account, one only has to choose 
the corresponding $\xi$ ratio given by the interpolation presented on Fig. 8.

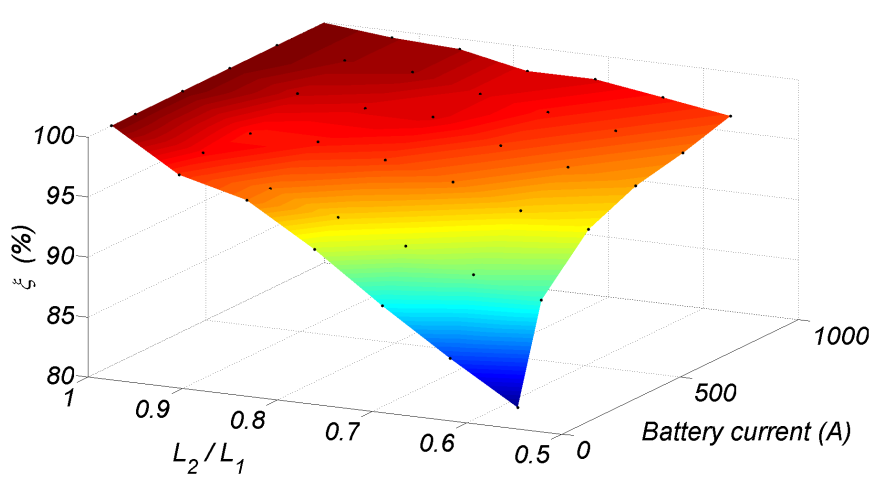

Fig. 8. Results of the $\xi$ coefficient expressed in $\%$ for different battery current and $L_{2} / L_{1}$ ratio.

\section{CONSIDERATION OF BOTH THE TWO OVERHANG EFFECTS: THE $2.5 \mathrm{D}_{\mathrm{COR}}$ MODEL}

In the two previous sections, the two different overhang effects were studied separately. In order to check the validity of the two developed modifications, both effects are now considered. The two previous models $\left(2.5 \mathrm{D}\right.$ and $\left.2 \mathrm{D}_{\mathrm{Cor}}\right)$ have been associated in a global single one and it is called $2.5 D_{\text {Cor }}$ model. The obtained results will be compared with $3 \mathrm{D}$ computations and measurements.

\section{A. Results vs. $3 D$ computations}

Now, $L_{1}$ and $L_{3}$ are considered to be constant and $L_{2}$ is a variable length. Therefore both $L_{2} / L_{1}$ and $L_{3} / L_{2}$ ratios change. The $2.5 \mathrm{D}_{\text {Cor }}$ model contains both the $e^{\prime}$ modified thickness of the yoke regarding the $L_{3} / L_{2}$ ratio, and the $\xi$ coefficient regarding the battery current and the $L_{2} / L_{1}$ ratio (obtained by interpolating the surface plot on Fig. 8). 3D finite element computations have been performed for four different $L_{3} / L_{2}$ and $L_{2} / L_{1}$ ratios, and four different batteries current. Results of the basic $2 \mathrm{D}$ model and the $2.5 \mathrm{D}_{\text {Cor }}$ model are compared with 3D calculations and displayed on Table II.

Results show that the $2.5 \mathrm{D}_{\text {Cor }}$ model is particularly close to the $3 \mathrm{D}$ computations whereas the non-modified 2D model has a relative error between $+11 \%$ and $-20 \%$ with the $3 \mathrm{D}$ model. The $2.5 \mathrm{D}_{\text {Cor }}$ model seems less accurate for the smallest $L_{2} / L_{1}$ ratio and low saturation levels. But even in this case, the maximal error is less than $6 \%$. In all the other cases, the error between the $3 \mathrm{D}$ and $2.5 \mathrm{D}_{\mathrm{Cor}}$ model is less or equal to $2.1 \%$.

Concerning the average CPU Time, the 3D model takes about 2 hours; our $2.5 \mathrm{D}_{\mathrm{Cor}}$ is around 15 seconds only. So, one can say that our model can retain the $3 \mathrm{D}$ accuracy and decreases strongly the total time (almost 1/500). Of course, this significant time gain must be nuanced in reminding that some 3D computations (needed to determine the $\xi$ factor as explained in part IV) have been previously performed before applying our $2.5 \mathrm{D}_{\text {Cor }}$ model. Nevertheless it permits to save a huge amount of time and let us solve some optimization problems as shown below.
TABLE II

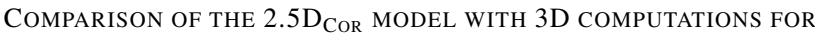
BOTH CUMULATIVE 3 D EFFECTS

\begin{tabular}{|c|c|c|c|c|}
\hline $\begin{array}{c}L_{3} / L_{2} \\
\text { ratio }\end{array}$ & $\begin{array}{c}l_{2} / L_{1} \\
\text { ratio }\end{array}$ & $\begin{array}{c}\text { Battery } \\
\text { current }(\mathrm{A})\end{array}$ & $\begin{array}{c}\Delta\left(\Gamma_{2 \mathrm{D}}, \Gamma_{3 \mathrm{D}}\right) \\
(\%)\end{array}$ & $\begin{array}{c}\Delta\left(\Gamma_{2.5 \mathrm{D}_{\mathrm{Cor}}}, \Gamma_{3 \mathrm{D}}\right) \\
(\%)\end{array}$ \\
\hline \multirow{4}{*}{1.85} & \multirow{4}{*}{0.93} & 200 & -7.5 & -1.6 \\
\hline & & 400 & -17.5 & -2.1 \\
\hline & & 600 & -20.1 & -0.9 \\
\hline & & 800 & -20.2 & 0.1 \\
\hline \multirow{4}{*}{2} & \multirow{4}{*}{0.86} & 200 & -2.5 & 0.1 \\
\hline & & 400 & -14.9 & -0.4 \\
\hline & & 600 & -18.2 & -0.2 \\
\hline & & 800 & -18.7 & 0.7 \\
\hline \multirow{4}{*}{2.18} & \multirow{4}{*}{0.79} & 200 & 3.4 & 2.5 \\
\hline & & 400 & -11.6 & 0.8 \\
\hline & & 600 & -15.9 & 1.1 \\
\hline & & 800 & -16.8 & 0.5 \\
\hline \multirow{4}{*}{2.40} & \multirow{4}{*}{0.71} & 200 & 10.7 & 5.8 \\
\hline & & 400 & -7.1 & 2.1 \\
\hline & & 600 & -12.6 & 0.6 \\
\hline & & 800 & -14.0 & 0.1 \\
\hline
\end{tabular}

\section{B. Results vs. measurements}

Since Fig. 3 shows the very good agreement of the 3D results and measurements, one can conclude that our $2.5 \mathrm{D}_{\mathrm{Cor}}$ model is much close to real experimental values. These conclusions are verified in Fig. 9 which compared mechanical torque measurements with a $2 \mathrm{D}$ classical model and our $2.5 \mathrm{D}_{\mathrm{Cor}}$ model.

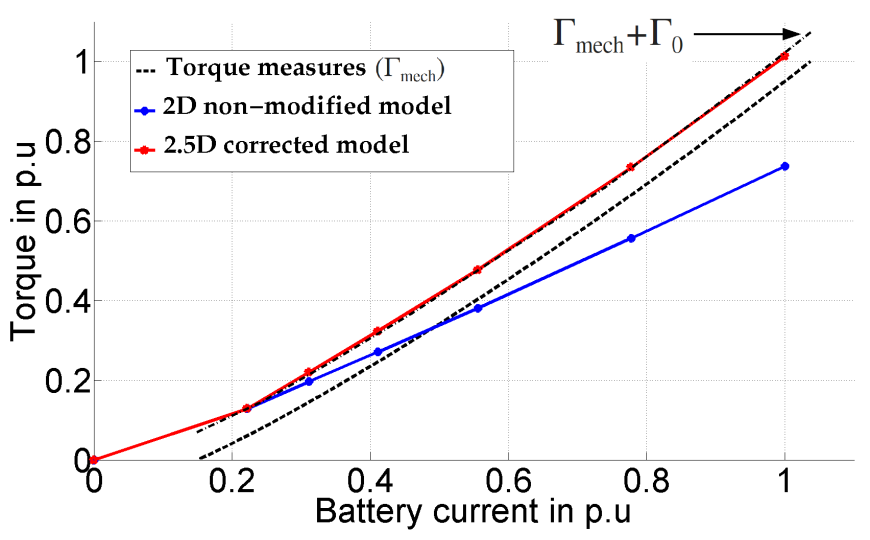

Fig. 9. Comparison between the two 2D models and measurements

If a translation on the mechanical measured torque $\left(\Gamma_{\text {mech }}\right)$ by a factor $\Gamma_{0}$ corresponding to the constant mechanical losses as explain in part II-C is performed, a really good agreement between the electromagnetic torque computed by the $2.5 \mathrm{D}_{\mathrm{Cor}}$ model and the experiment can be noticed.

\section{OPTIMAL DESIGN EXAMPLES}

The developed $2.5 \mathrm{D}_{\text {Cor }}$ model is quite easy to implement and use with an optimization algorithm. To illustrate this, some examples of optimal design will be carried out in the following. 


\section{A. Design goals}

As it is mentioned in the introduction, two solutions may satisfy the requirements of the micro-hybrid function: a startergenerator or even just a reinforced starter. The present work focus on the second one. Its main advantages are its robustness and its comparatively low cost, whereas its main drawbacks are a relatively low efficiency and high noise.

For such devices, there are two really important objectives: the compactness and the lifetime. For the first one, it can be satisfied by minimizing the global external volume of the starter. For the second one, a rational way consists in reducing the brush wear by decreasing the peripheral speed of collector at the operating point. This is why it is relevant (suitable) to maximize the electromagnetic torque.

The performances such as the efficiency, or the noise may be improved, but in our application the battery current must be limited in order to keep a small voltage drop. Consequently the value of the electrical resistance of the device is imposed, and then the Copper losses too. To reduce the core losses is not also particularly relevant because they represent a small fraction of the total losses (around 5\%).

Consequently, for our application, good approaches are those which minimize the external volume for a given torque, or maximize the torque in a given volume. The following examples cover both or one of these aspects.

\section{B. Variables definition and reference solution}

All of our design problems deal with the same variables, which are:

- 5 real variables: the length of the armature, the armature diameter, the iron yoke thickness, the pole shoe width and the wire cross section of the armature windings.

- 3 integer variables: the kind of flat cable around the pole, the number of turns around the pole, and the number of slot in the armature.

- 3 non-linear constraints: one equality on the total electric resistance, and two geometric relations traducing the constructability of the device.

All these variables and their corresponding bounds are listed in Table III and form our unknown vector $\mathbf{x}$.

TABLE III

VARIABLES OF THE CONSIDERED DESIGN PROBLEM.

\begin{tabular}{c|c|c} 
Symbol & Quantity & Bounds \\
\hline \hline$L_{1}$ & length of the armature & {$[25,50] \mathrm{mm}$} \\
$D_{a}$ & diameter of the armature & {$[45,55] \mathrm{mm}$} \\
$t_{y}$ & thickness of iron yoke & {$[3,6.5] \mathrm{mm}$} \\
$w_{p}$ & width of pole shoe & {$[12,19] \mathrm{mm}$} \\
$d_{a}$ & diameter of armature windings wire & {$[2.7,3.7] \mathrm{mm}$} \\
$\sigma_{f w}$ & kind of field windings flat cable & $\llbracket 1,124 \rrbracket$ \\
$N_{f w}$ & number of turns in field winding & $\llbracket 8,12 \rrbracket$ \\
$N_{s}$ & number of armature slots & $\{17,19,21,23\}$ \\
\hline
\end{tabular}

In order to quantify the possible improvement of our optimal solutions, the reinforced starter used for the experimental validations in previous section is chosen. In fact, this particular device correspond to our state of the art. In the following, its external volume will be called $V_{\text {ref }}$ and its electromagnetic torque $\Gamma_{\text {ref. }}$.

\section{Mono-objective problems}

Firstly each objective will be considered separately. Then, two kinds of problems can be defined, two particular cases $(P 1)$ and $(P 2)$, given by (8) and (9) will be solved:

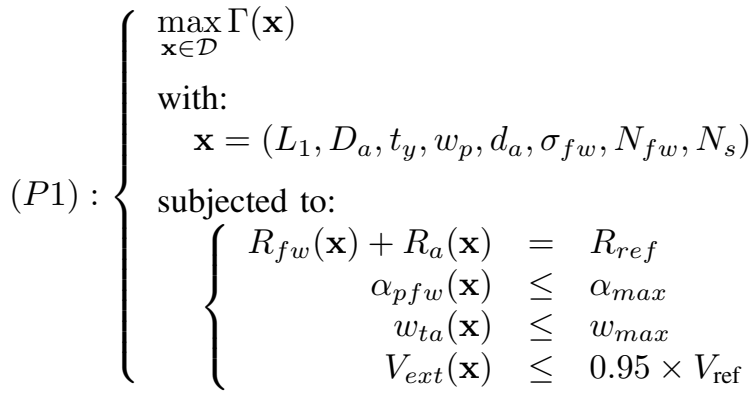

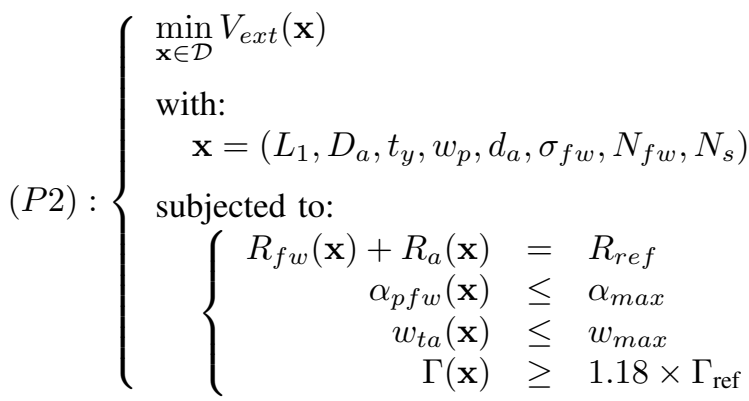

Where $R_{f w}$ and $R_{a}$ are respectively the electrical resistance of the field windings and the armature windings, $\alpha_{p f w}$ is the angular length of a complete field winding, and $w_{t a}$ is the width of a teeth in the armature.

A particle swarm algorithm (PSO) has been chosen to solve these problems because of its simplicity of implementation and its relatively good performance [16]. Our own code which can handle integer variables as $\sigma_{f w}, N_{f w}$ and $N_{s}$ is used. Convergence of the algorithm and obtained results are displayed on Fig. 10. The runs are made with 40 individuals moving during 100 iterations. Results are given in per unit to facilitate comparison; it means that if the torque is equal to 1 its value is $\Gamma_{\text {ref }}$, and for the external volume 1 correspond to $V_{\text {ref. }}$.

Each run takes about 8 hours of computation, whereas if a 3D model had been used, the optimization process would have needed a CPU time of around 330 days. It is clearly shown that the both obtained solutions are better than our reference. For $(P 1)$, at the end of the process a device with a slightly greater torque and with a volume less than $0.95 \times V_{\text {ref }}$ (since our all constraints are satisfied) is found. The second solution is even more relevant because it increases the torque by $18 \%$ $\left(1.18 \times \Gamma_{\text {ref }}\right)$ and only by $5 \%$ the external volume.

Results show that our model coupled with a mono-objective optimization process can provide quickly good optimal solutions, better than our state of the art. However, as explained above, it is the compromise torque vs. external volume which is the most interesting for us. This is why we deal with a multi-objective problem hereafter. 


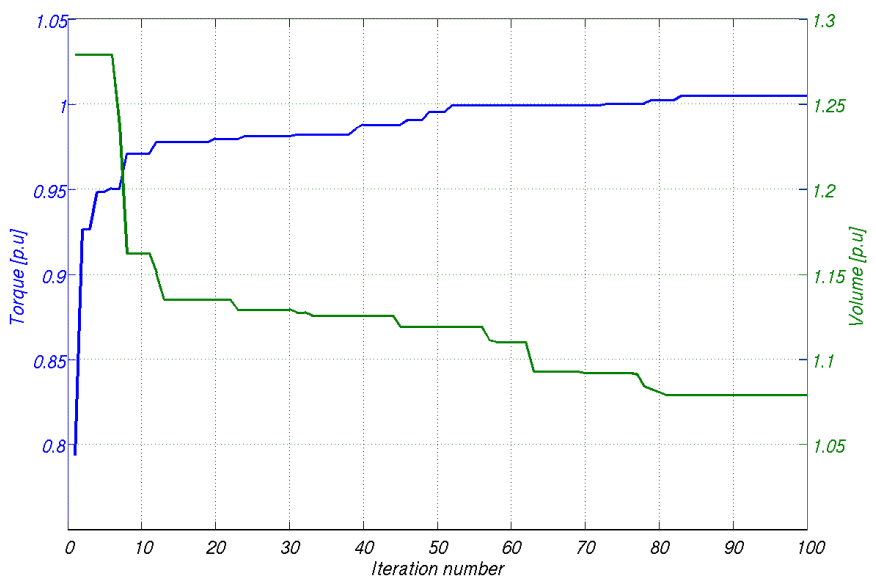

Fig. 10. Convergence of the mono-objective optimization processes for $(P 1)$ (in blue) and ( $P 2)$ (in green).

\section{Multi-objective problem}

The problem consists in both minimizing the total external volume of the starter and maximizing its electromagnetic torque. Thus, a two-objective problem with 8 variables under 3 non-linear constraints is solved, its mathematical formulation is given by:

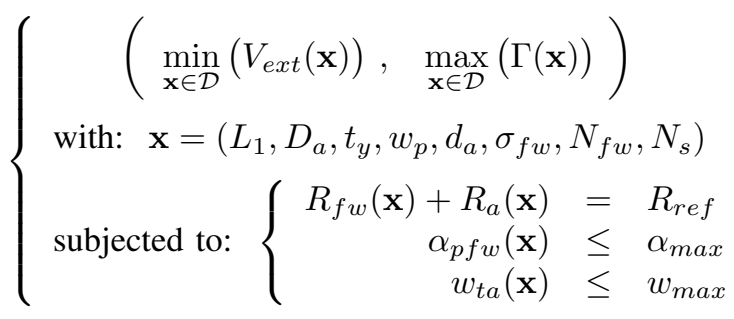

The selected algorithm is a Non-dominated Sorting Genetic Algorithm NSGA-II, because it is widely used, even recently [17], [18], and obtains generally good performances on multiobjective problems [19]. A typical implementation modified in order to take into account mixed variables is used, as in [20]. The problem was solved with 40 individuals evolving during 100 generations. After 8 hours of computations for the whole process, the pareto front given in Fig. 11 is obtained (results are again displayed in per unit as above).

Firstly, the CPU time of the problem resolution is nearly the same than in the mono-objective cases since the same numbers of individuals and iterations have been chosen. The CPU time is a thousand times smaller than if a 3D model coupled with the NSGA-II algorithm had been used.

On Fig. 11, our state of the art solution (coordinates $(1 ; 1)$ ) and the iterations corresponding to the two mono-objective problems $(P 1)$ and $(P 2)$ (respectively the red and purple broken lines) are also reported. All the solutions given by the Pareto front clearly dominate our state of the art. It also appears that both previous mono-objective solutions converge towards this front but are also dominated. This confirms the efficiency of the multi-objective process. Another fact of interest is that the obtained optimal solutions which have an external volume close to our reference but a higher torque have all a number of slots in the armature $N_{s}$ equal to 17. It is a

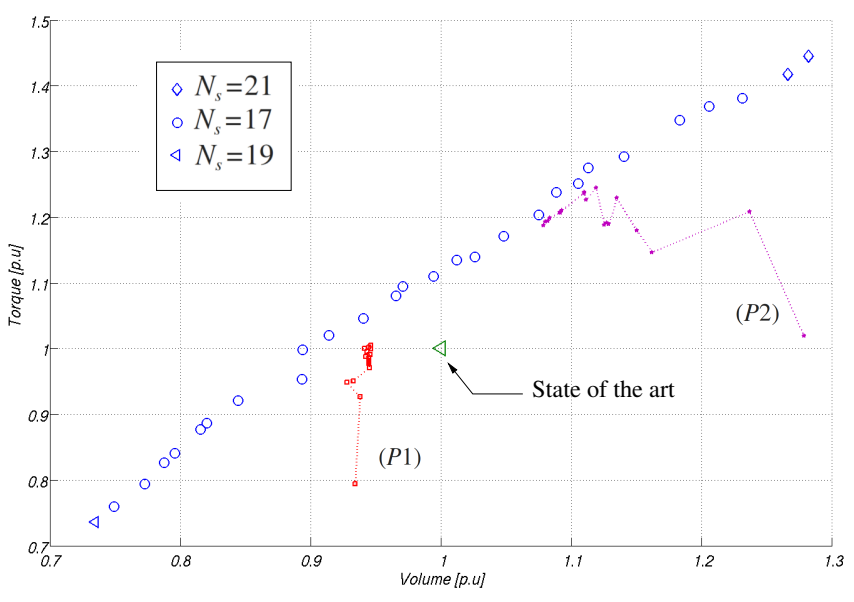

Fig. 11. Pareto front of the torque vs. the external volume (in p.u).

bit surprising because our reference correspond to $N_{s}=19$, and in a preliminary approach, we could think to rise this number in order to increase the torque. This can be explained by the fact that in the $N_{s}=17$ case, the magnetic circuit of the armature is best used and the teeth are less saturated. It shows that thanks to the multi-objective optimization we can obtained really more information rather than mono-objective cases but with the same CPU times.

The goal of the present examples is to show that the $2.5 \mathrm{D}_{\text {Cor }}$ model allows to solve effectively hard optimization problems of reinforced starters design. Our methodology have been successfully applied and proves to be a really efficient tool to improve reinforced starter design.

\section{CONCLUSION}

In the context of energy saving in automotive industry, the reinforced starter may stay a good solution for the stop-start topology. This device can optimized and fulfill the requirements of this application in terms of life-time. Due to its low cost and simplicity of supply, it should be a competitive solution compared to the other solutions using different other kinds of electric machines.

The optimization of such devices needs to take into account the strong 3D effects due the geometry. In this paper, the authors have proposed a 2D model modified and adapted to account for two important 3D effects in the machine. The model is based on iterating two 2D finite element computations for the yoke vs. pole shoes overhang characterization. This method gives similar results to 3D calculations, with little CPU time consumption, in comparison. The armature vs. pole shoes overhang is identified using a DOE method based on 3D finite element calculations. Finally, those two overhang effects are included into a $2 \mathrm{D}$ finite element model called $2.5 \mathrm{D}_{\text {Cor }}$ model. This model has been validated by both experimental results and 3D finite element computations.

This new model is accurate enough to consider an optimization process of the device which could be difficult or impossible with a 3D finite element model because of high CPU time. It has been used in mono-objective and multiobjective optimization processes of a starter under strong 
constraints of different types. Good results have been obtained by mean of very reasonable CPU time when compared to the use of full 3D model.

\section{REFERENCES}

[1] "Regulation (EC) No 443/2009 of the European Parliament and of the Council of 23 April 2009 setting emission performance standards for new passenger cars as part of the Community's integrated approach to reduce CO 2 emissions from light-duty vehicles," Official Journal of the European Union, pp. L140/1-L140/15, 2009.

[2] A. Vauquelin, J-P. Vilain, S. Vivier, N. Labbe, B. Dupeux, "A new modeling of DC machine taking into account commutation effects," Proc. ICEM 2008, 18th International Conference on Electrical Machines, pp.16, 2008.

[3] R. Andreux, J. Fontchastagner, N. Takorabet, N. Labbe, J-S. Metral, "Magnetic Field-Electric Circuit Coupled Method for Brush DC Motor Simulations", Proc. ICEM 2012, 20th International Conference on Electrical Machines, 2012.

[4] K-Y Hwang, H. Lin, S-E Rhyu, B-I K, "A Study on the novel Coefficient Modelling for Modelling a Skewed Permanent Magnet and Overhang structure for Optimal Design of Brushless DC Motor," IEEE Trans. Magn., vol. 48, no. 5, pp.1918-1923, 2012.

[5] K-C. Kim, D-H. Koo, J. Lee, "The study on the overhang coefficient for permanent magnet machine by eperimental design method," IEEE Trans. Magn., vol. 43, no. 6, pp.2483-2485, 2007.

[6] Y-D. Chun, J. Lee, "Overhang Effect Analysis of Brushless DC Motor by 3-D Equivalent Magnetic Circuit Method," IEEE Trans. Magn., vol. 39, no. 3, pp.1610-1613, 2003.

[7] B. Funieru, A. Binder, "Simulation of Electrical Machines end Effects with reduced Length 3D FEM Models," Proc. ICEM 2012, 20th International Conference on Electrical Machines, 2012.

[8] K-C. Kim, J. Lee, "The Dynamic Analysis of a Spoke-Type Permanent Magnet Generator With Large Overhang," IEEE Trans. Magn., vol. 41, no. 10, pp.3805-3807, 2005.

[9] Z. Zhu, J.T. Chen, Y. Pang, D. Howe, S. Iwasaki, R. Deodhar, "Modeling of End-Effect in Flux-Switching Permanent magnet Machines," Proc. ICEMS, pp.943-948, 2007.

[10] I. Tsukerman, V. Dombrovski, "Finite-Element Simulation of TimeDependent Electromagnetic Fields in the End Zone of Superconducting Motors," IEEE Trans. Magn., vol. 38, no. 2, pp.1265-1268, 2002.

[11] J. Jamali, "End effects in linear induction and rotating electrical machines," IEEE Trans. Energy Convers., vol. 18, pp.440-447, 2003.

[12] H. Baernklau and T. Bach, "Experience with model simplifications for making a large FEM calculation case computable," Proc. ICEM 2008, 18th International Conference on Electrical Machines, pp.1-5, 2008.

[13] Byeong-Hwa Lee, Jung-Pyo Hong and Jung-Ho Lee, "Optimum Design Criteria for Maximum Torque and Efficiency of a Line-Start PermanentMagnet Motor Using Response Surface Methodology and Finite Element Method," IEEE Trans. Magn., vol. 48, no. 2, pp. 863-866, 2012.

[14] Jung-Ho Lee, "Optimum Shape Design Solution of Flux Switching Motor Using Response Surface Methodology and New Type Winding," IEEE Trans. Magn., vol. 48, no. 4, pp.1637-1640, 2012.

[15] Song Xiao, M. Rotaru and J. K. Sykulski, "Adaptive Weighted Expected Improvement With Rewards Approach in Kriging Assisted Electromagnetic Design," IEEE Trans. Magn., vol. 49, no. 5, pp.2057-2060, 2013.

[16] Bin Xia, Minh-Trien Pham, Yanli Zhang and Chang-Seop Koh, "A Global Optimization Algorithm for Electromagnetic Devices by Combining Adaptive Taylor Kriging and Particle Swarm Optimization," IEEE Trans. Magn., vol. 49, no. 5, pp.2061-2064, 2013.

[17] L.G. Campana, P. Di Barba, F. Dughiero, C.R. Rossi and E. Sieni, "Optimal Needle Positioning for Electrochemotherapy: A Constrained Multiobjective Strategy," IEEE Trans. Magn., vol. 49, no. 5, pp.21412144, 2013.

[18] T.C. Bora, L. Lebensztajn and L.D.S. Coelho, "Non-Dominated Sorting Genetic Algorithm Based on Reinforcement Learning to Optimization of Broad-Band Reflector Antennas Satellite," IEEE Trans. Magn., vol. 48, no. 2, pp.767-770, 2012.

[19] Gang Lei, X.M. Chen, J.G. Zhu, Y.G. Guo, Wei Xu and K.R. Shao, "Multiobjective Sequential Optimization Method for the Design of Industrial Electromagnetic Devices," IEEE Trans. Magn., vol. 48, no. 11, pp.4538-4541, 2012.

[20] J. Le Besnerais, V. Lanfranchi, M. Hecquet and P. Brochet, "Multiobjective Optimization of Induction Machines Including Mixed Variables and Noise Minimization," IEEE Trans. Magn., vol. 44, no. 6, pp.1102-1105, 2008. 